REGARDS

SUR LECONOMIE ALLEMANDE

BULLETIN ECONOMIQUE DU CRAC

\section{Regards sur l'économie allemande}

Bulletin économique du CIRAC

$101 \mid 2011$

Varia

\title{
Le système bancaire allemand et la crise financière
}

\section{Patrick Brämer, Horst Gischer et Toni Richter}

Traducteur : Isabelle Bourgeois

\section{CpenEdition}

\section{Journals}

Édition électronique

URL : http://journals.openedition.org/rea/4285

DOI : $10.4000 /$ rea. 4285

ISBN : 978-2-8218-0954-3

ISSN : 1965-0787

Éditeur

CIRAC

Édition imprimée

Date de publication : 10 juin 2011

Pagination : 5-16

ISSN : 1156-8992

\section{Référence électronique}

Patrick Brämer, Horst Gischer et Toni Richter, « Le système bancaire allemand et la crise financière »,

Regards sur l'économie allemande [En ligne], 101 | juin 2011, mis en ligne le 01 juin 2013, consulté le 19 avril 2019. URL : http://journals.openedition.org/rea/4285 ; DOI : 10.4000/rea.4285 


\section{Le système bancaire allemand et la crise financière}

\section{Patrick Brämer, Horst Gischer et Toni Richter}

La récente crise de la finance mondiale a laissé une profonde empreinte. Et les discussions sur les mesures les plus adéquates à adopter pour éviter que de tels dysfonctionnements se reproduisent à l'avenir sont en cours. Mais si on excepte les premières ébauches, telles qu'elles se dessinent par exemple dans le cadre des accords du G20, rien ne permet de déceler un progrès significatif dans la régulation du marché (mondial) de la finance ni non plus dans la réforme pourtant indispensable des systèmes financiers. A maintes reprises, par le passé, le système bancaire allemand s'était attiré les critiques pour sa prétendue inefficience et la cœxistence des banques privées, de caisses d'épargne (Sparkassen) et de banques coopératives (Genossenschaftsbanken), considérées comme dommageable à sa productivité (voir par exemple Brunner et al., 2004 ; Sachverständigenrat, 2008). Or dans la situation actuelle, ce "système à trois piliers" s'est révélé facteur de stabilité. Car c'est justement l'existence de toutes ces 'petites entités' si souvent décriées qui a protégé le système bancaire allemand contre une crise systémique de grande ampleur. Face aux turbulences que traverse le marché de la finance mondiale, il paraît judicieux de détailler le rôle et les missions du secteur public au sein d'un système bancaire à la structure polycentrique afin de rendre ses spécificités plus compréhensibles aux lecteurs peu familiers des réalités allemandes. II convient également de mieux identifier les fonctions macro-économiques qu'exercent les Sparkassen à la différence des autres acteurs en présence, qui soit ne les remplissent pas, soit ne les exercent pas de leur propre initiative dans une ampleur comparable.

\section{Le « système à trois piliers ॥ : structure et évolution}

Le système bancaire allemand compte environ 2000 établissements de crédit au statut juridique autonome et dont l'écrasante majorité exerce les différents métiers de la banque universelle, centrés sur les activités classiques d'intermédiation. De ce fait, au sein de la structure à trois piliers née au fil de l'histoire, le système bancaire allemand se distingue de celui des autres Etats de l'UE. Avec une part de plus du tiers $(36,2 \%)$ du total de l'actif des banques, tous types confondus, les banques commerciales constituent le premier pilier. Le deuxième, avec une part (cumulée) de 30,5\%, est celui des établissements de droit public (öffentlich-rechtlich). Quant aux banques coopératives (Genossenschaftsbanken), elles forment le troisième pilier, mais avec une part nettement inférieure (en ce qui concerne le total des actifs).

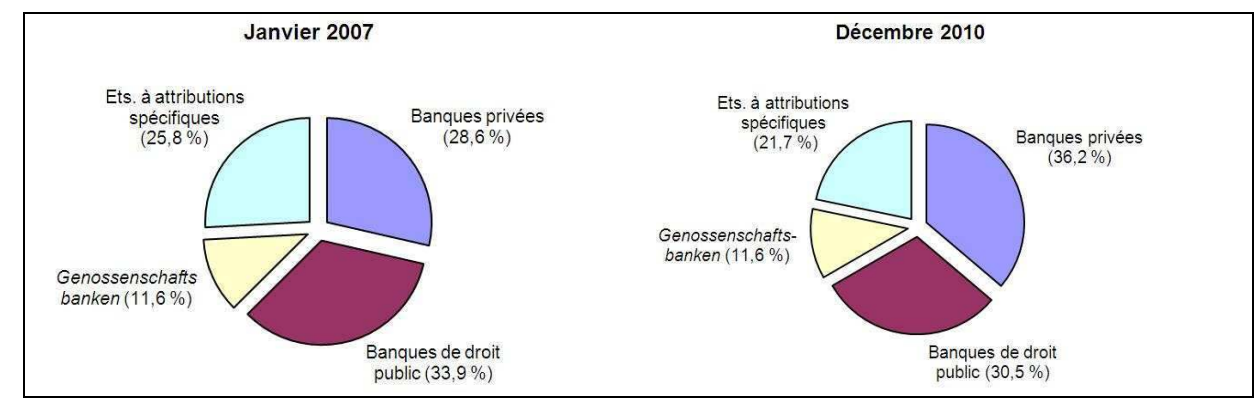

Source des données : Deutsche Bundesbank, calculs des auteurs.

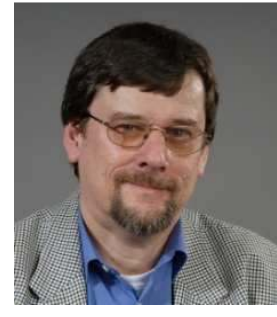

Horst Gischer professeur d'économie et de finance, est titulaire de la chaire d'économie (monnaie et crédit) à l'Otto-GuerickeUniversität (Magdeburg)

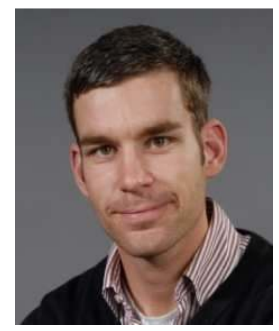

Patrick Brämer, collaborateur scientifique, est diplômé d'études commerciales

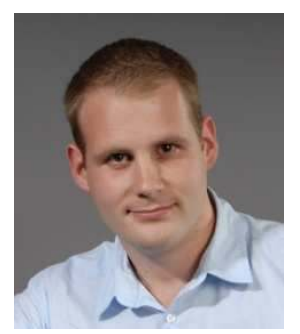

Toni Richter, collaborateur scientifique, est diplômé d'études économiques

La part des différents secteurs dans l'actif total du système bancaire allemand est restée stable 
Comme le révèle le graphique ci-dessus, la crise financière n'a apporté aucun changement dans la structure à trois piliers du système bancaire allemand. On constate par ailleurs la place non négligeable qu'y occupe le groupe des établissements de crédit à attributions spécifiques qui réunit les banques hypothécaires, les caisses d'épargne-logement (Bausparkassen), les banques directes comme les établissements dotés de missions particulières (ex. : la KfW).

Tableau 1 : La structure du système bancaire allemand à trois piliers

\begin{tabular}{|c|c|c|c|c|c|}
\hline & \multicolumn{2}{|c|}{ Nombre d'établissements } & \multicolumn{2}{|c|}{ Nombre de succursales } & \multirow{2}{*}{$\begin{array}{l}\text { Mission ou objectif } \\
\text { commercial }\end{array}$} \\
\hline & 2007 & 2010 & 2007 & 2010 & \\
\hline $\begin{array}{l}\text { Banques privées } \\
\text { - Grandes banques commerciales }\end{array}$ & $\begin{array}{c}360 \\
5\end{array}$ & $\begin{array}{c}288 \\
4\end{array}$ & $\begin{array}{l}11578 \\
8879\end{array}$ & $\begin{array}{l}11496 \\
8773\end{array}$ & Maximalisation des bénéfices \\
\hline $\begin{array}{l}\text { Ets. de droit public } \\
\text { - Sparkassen } \\
\text { - Landesbanken }\end{array}$ & $\begin{array}{r}469 \\
457 \\
12\end{array}$ & $\begin{array}{r}441 \\
431 \\
10\end{array}$ & $\begin{array}{r}14252 \\
13756 \\
496\end{array}$ & $\begin{array}{r}13741 \\
13266 \\
475\end{array}$ & $\begin{array}{l}\text { Mission d'intérêt général, } \\
\text { service public }\end{array}$ \\
\hline $\begin{array}{l}\text { Banques coopératives } \\
\text { - Coopératives de crédit } \\
\text { - Organismes centraux }\end{array}$ & $\begin{array}{r}1261 \\
1259 \\
2\end{array}$ & $\begin{array}{r}1162 \\
1160 \\
2\end{array}$ & $\begin{array}{r}12594 \\
12583 \\
11\end{array}$ & $\begin{array}{r}12155 \\
12144 \\
11\end{array}$ & $\begin{array}{c}\text { Promotion de l'activité } \\
\text { économique des membres, } \\
\text { à but lucratif }\end{array}$ \\
\hline
\end{tabular}

Source des données : Deutsche Bundesbank.

Banques privées Le groupe des établissements de crédit compte, outre les grandes banques commerciales, les banques régionales, les petites banques privées, ainsi que les succursales des banques étrangères. L'objectif commercial premier des 288 établissements de crédit recensés en 2009, généralement des sociétés par actions, est de réaliser des bénéfices. Du fait du profil majoritairement international de leur clientèle d'affaires, la part des opérations réalisées à l'étranger et celle des opérations sur titres est plus importante dans leurs activités que dans les établissements des deux autres piliers (voir Hartmann-Wendels et al., 2010, p. 28 sq.).

Banques coopératives

Le groupe des banques coopératives comprend 1160 coopératives de crédit, ainsi que leurs deux organismes centraux. Créées avec pour mission expresse de "promouvoir la prospérité et l'activité de leurs membres (sociétaires)" (ibid., p. 34), ces banques n'en cherchent pas moins avant tout à générer des profits. Certes, leur clientèle s'est élargie et diversifiée, et elles offrent la gamme complète de leurs produits (des opérations de dépôt aux crédits) à tous leurs clients, qu'ils soient membres ou non. Néanmoins, le cœur de clientèle des $\mathrm{Ge}$ nossenschaftsbanken se compose toujours des ménages et des PME. Et, bien qu'il ne soit pas juridiquement contraignant, elles sont restées fidèles au principe de la limitation régionale de leur rayon d'action (voir Stiele, 2008, p. 30).

Etablissements de droit public : Sparkassen et Landesbanken

Le troisième pilier - celui des établissements bancaires de droit public - regroupe d'un côté les 438 Sparkassen et, de l'autre, les 9 banques régionales (Landesbanken). Avec plus de 13700 succursales, les caisses d'épargne constituent le réseau le plus dense, tous secteurs confondus, et qui couvre l'ensemble du territoire, des zones urbaines aux régions rurales. A l'inverse des banques commerciales privées, les banques universelles de droit public que sont les Sparkassen placent expressément leur stratégie commerciale au service de l'intérêt général et remplissent une " mission de service public » (öffentlicher Auftrag ; voir encadré 1).

Alors que le domaine de compétences des Landesbanken dépasse leur fonction originelle de banques centrales ou d'office de compensation des Sparkassen implantées dans leur région et inclut des transactions plus complexes sur les marchés, des transactions internationales et des opérations de crédit dont le volume dépasse les capacités d'une Sparkasse isolée, le cœur de métier des Sparkassen est l'intermédiation bancaire classique, principalement les opérations de crédit basées sur les opérations de dépôt. Tout comme la clientèle des banques coopératives, celle des caisses d'épargne se compose essentiellement de ménages comme de petites et moyennes entreprises (voir Hackethal/ Schmidt, 2005, p. 133 sq.). 


\section{Encadré 1 : Les particularités du secteur bancaire de droit public en Allemagne}

\section{Porteurs et garants}

Le secteur bancaire de droit public comprend d'une part les Sparkassen, dont les 'propriétaires' ou 'porteurs' et garants (Träger) sont, à de rares exceptions près, des communes, des administrations, des districts régionaux ou des syndicats communaux et, de l'autre, les Landesbanken dont le capital est détenu la plupart du temps par un Land, les fédérations des caisses d'épargne, et d'autres Landesbanken. II compte actuellement plus de 440 établissements de type banque universelle et qui exercent donc (et sont habilitées à le faire) les mêmes activités (transactions actives et passives) que leurs concurrents du privé. S'ajoute, à la tête de ce groupe, la Deka-Bank, un établissement détenu (pour l'instant encore) à parité par les Landesbanken et les Sparkassen. Leur statut de droit public (öffentlich-rechtlich) interdit à ces établissements d'être rachetés par des banques privées; mais il leur réserve la possibilité d'entrer au capital d'établissements de droit privé.

\section{«Mission de service public »}

L'idée directrice qui préside aux missions de toutes les banques au statut " d'établissement et collectivité de droit public » (Anstalt und Körperschaft des öffentlichen Rechts) est celle d'intérêt général ou de service public (voir Brämer et al., 2010a).

Les Sparkassen, le socle de ce service public, sont dotées de la mission expresse d'assurer un approvisionnement en services financiers et en crédit accessible à tous, ce qui implique d'entretenir un réseau de succursales couvrant l'ensemble du territoire (voir tableau 1). A cela s'ajoute l'obligation légale de satisfaire aux besoins en crédit de l'économie de la région d'implantation - en particulier des PME -, de promouvoir l'esprit d'épargne, la constitution du patrimoine, et de soutenir le développement d'un marché concurrentiel dans les régions à faible dynamisme économique. En outre, grâce à leur stratégie axée sur l'économie réelle, elles doivent exercer un effet stabilisateur sur l'ensemble du système financier allemand (voir ibid., $p$. 328).

La mission première des Landesbanken, qui constituent un deuxième échelon dans le système des caisses d'épargne, est de centraliser les fonctions d'offices de compensation et de refinancement. En qualité de banques centrales de virements des caisses d'épargne (Girozentralen), elles assurent essentiellement les transactions par chèques et virements pour le compte des Sparkassen (caisses locales), gèrent leurs avoirs liquides et leur octroient des avances bancaires de trésorerie (voir Brämer et al., 2010b).

Graphique 2 : Structure du secteur bancaire public

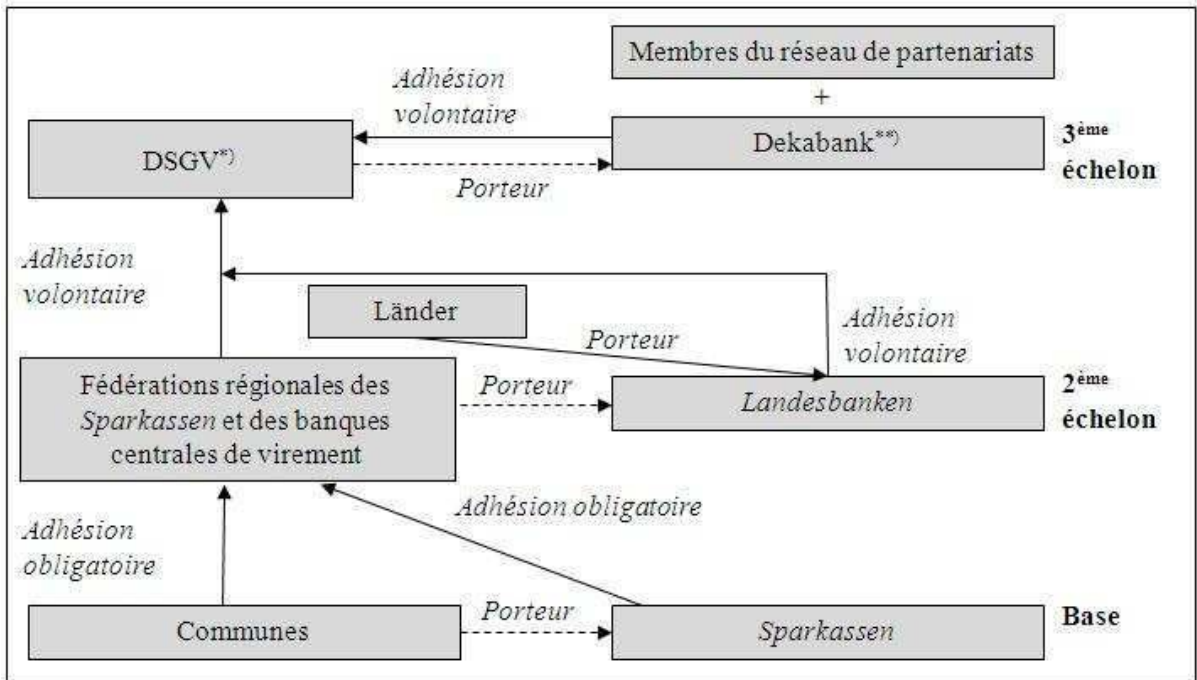

Source : graphique des auteurs, d'après Tolkmitt, 2007 (p. 57). *) DSGV : Deutscher Sparkassen- und Giroverband, Association allemande des caisses d'épargne et Landesbanken. $\left.{ }^{* *}\right)$ Banque centrale de virements du réseau des Sparkassen (Deutsche Girozentrale).

\section{Les principes fondateurs d'un système organisé en réseau}

Ces domaines d'activité traditionnels des Landesbanken sont intimement liés à deux principes normatifs au fondement de la 'philosophie' de réseau qui caractérise les caisses d'épargne allemandes : le principe de la région d'implantation (Regionalprinzip) et le principe de subsidiarité. Ils sont tous deux ancrés dans les lois des Länder sur les Sparkassen.

Le principe régional se traduit par une limitation géographique de leur action à une zone d'activités locale ou régionale donnée (voir Frankenberger, 2004, p. 14 sq.). L'idée qui sous-tend ce principe est d'interdire la prise de tout risque hors du territoire des porteurs et garants d'une caisse dépargne, et qui serait de ce fait difficile à évaluer. Par ailleurs, cette forme de structu- 
ration horizontale vise à éviter la concurrence intra-sectorielle entre établissements de même niveau. Ce principe d'organisation structurelle et de régulation (Ordnungsprinzip) s'applique jusqu'à aujourd'hui à la base du groupe Sparkassen-Finanzgruppe, se limitant toutefois aux opérations de dépôt des Sparkassen.

De nature plus abstraite à première vue est le principe de subsidiarité qui caractérise le partage des rôles au sein du réseau. II régit la répartition des compétences entre les différents échelons au sein de la Sparkassen-Finanzgruppe: il revient ainsi en premier à la plus petite entité, c'est-à-dire la Sparkasse locale, de remplir toutes les missions qu'il lui est possible d'exercer pleinement et en toute autonomie. Ce n'est que lorsque celle-ci atteint les limites légales ou économiques de son action qu'intervient l'échelon supérieur. En conséquence, la délégation de certains domaines d'activité ou tâches aux Landesbanken s'effectue de façon subsidiaire, c'est-à-dire du bas vers le haut, de l'échelon inférieur à l'échelon supérieur.

Cette proximité géographique à la base entre la caisse d'épargne (intermédiation) et son client, qui est le corollaire du principe de verticalité dans l'organisation, contribue à réduire l'asymétrie de l'information typique des marchés bancaires et permet à la Sparkasse locale de décider en pleine autonomie, en toute connaissance de cause et en temps réel de l'octroi de crédit à ses clients (voir Gischer et al., 2005, p. 69 sq.). Quant à la coopération entre les établissements à l'échelon supérieur au sein du réseau, elle permet de garantir l'efficience des activités de chacun de ses membres. Ces deux principes (le principe de subsidiarité et celui de la région d'implantation) se concrétisent dans la structure à trois niveaux de l'organisation des Sparkassen, née au fil de l'histoire et qui s'est maintenue jusqu'à aujourd'hui. Le graphique 2 ci-dessus, qui présente leur organisation actuelle, retrace en outre la structure dualistique particulière du financement ou de la proriété (Trägerschaft) qui est elle aussi le reflet de l'imbrication des divers échelons (voir Schackmann-Fallis/Weiß, 2010, p. 84).

\section{La crise financière et le modèle économique des banques allemandes}

Les plus affectées : les banques commerciales...
Bien que la crise de la finance mondiale ait affecté le système bancaire allemand dès le printemps 2007, il n'est pas encore possible, même en 2011, d'évaluer définitivement et dans toute leur ampleur les dysfonctionnements induits tant sur des secteurs particuliers que sur l'ensemble de l'économie. Et dans ce contexte, affirmer que le secteur bancaire allemand dans son ensemble a bien résisté à la crise n'a guère de sens. II est plus pertinent de considérer isolément chacune des catégories de banques. Leur analyse révèle en effet que la crise de la finance mondiale a eu des impacts très différents selon les types d'établissements. Les catégories qui ont dû affronter une hausse des amortissements et des provisions pour dépréciation et dû recourir aux mesures de soutien de l'Etat sont essentiellement celles dont les modèles économiques se caractérisent par une internationalisation croissante et un engagement accru dans le métier de la banque d'investissement. A l'opposé, les établissements aux activités centrées sur leur région et non prioritairement axées sur la maximisation des profits ont de toute évidence traversé la période des tensions mondiales sans voir leur existence mise en danger.

Tableau 2 : Résultat avant impôts (en millions $€$ )

\begin{tabular}{|lrrrrr|}
\hline & $\mathbf{2 0 0 5}$ & $\mathbf{2 0 0 6}$ & $\mathbf{2 0 0 7}$ & $\mathbf{2 0 0 8}$ & $\mathbf{2 0 0 9}$ \\
\hline Tous secteurs confondus & 33242 & 27597 & 20531 & -25014 & -2870 \\
Etablissements de crédit privés & 17948 & 10144 & 18726 & -16420 & -6493 \\
- grandes banques commerciales & 14867 & 7520 & 15290 & -17833 & -6691 \\
Landesbanken & 3030 & 6014 & 788 & -6051 & -5229 \\
Sparkassen & 4927 & 4421 & 3759 & 2161 & 4713 \\
Coopératives de crédit & 4156 & 3614 & 2880 & 2039 & 3410 \\
\hline
\end{tabular}

Source des données : Deutsche Bundesbank.

... et les Landesbanken

L'évolution des bilans des principales catégories de banques allemandes avant et après la crise (tableau 2) confirme ce constat. Les résultats des exercices 2008 et 2009 révèlent que ce sont les établissements de crédit privés et les Landesbanken de droit public qui ont été particulièrement affectés par la crise financière. Durant la seule année 2008, ces deux catégories ont contribué respectivement à hauteur de près de -35 milliards $€$ et de plus de -7 milliards $€$ 
au recul (plus de 45 milliards $€$ par rapport à 2007) du résultat avant impôts des banques, tous secteurs confondus. Les Sparkassen et les coopératives de crédit ont certes enregistré elles aussi d'importantes pertes, mais globalement, leurs bilans restent excédentaires en moyenne. En 2009 également, le résultat avant impôts reste négatif, tant pour l'ensemble du secteur bancaire que pour les banques privées et les Landesbanken. Mais seules les Sparkassen et les coopératives de crédit ont pu dépasser dès 2009 leur niveau de 'résultats d'avant la crise'. Leurs modèles économiques, souvent décriés comme passés de mode ou peu originaux, s'étaient montrés particulièrement résistants aux violentes turbulences sur les marchés financiers (voir Brämer et al., 2011). A l'inverse, les stratégies poursuivies par les Landesbanken, les banques privées et les établissements à attributions particulières (comme par exemple la Hypo Real Estate), qui avaient multiplié les engagements sur les marchés des capitaux à l'international, avaient révélé d'importantes faiblesses.

\section{Des modèles économiques différents}

Pour mieux appréhender les répercussions de la crise financière sur les différents secteurs, il convient de considérer au préalable les différences foncières entre les modèles économiques des diverses catégories de banques. Dans un premier temps, une typologie plus précise de ces catégories fait apparaître (voir tableau 3) que les Landesbanken et les grandes banques commerciales, qui totalisent environ $20 \%$ de l'actif bancaire, ont une part importante sur le marché bancaire allemand. Indépendamment de cela, durant la crise, la répartition des parts de marché s'est légèrement modifiée au profit des grandes banques privées. Par ailleurs, ces dernières et les Landesbanken se caractérisent par un fort volume d'activités dans le segment des opérations financières interbancaires : ces deux groupes ont chacun à son actif un cinquième des créances bancaires en 2010 et détiennent proportionnellement une part similaire des dépôts bancaires. Le constat est pratiquement identique pour le début de l'année 2007.
Grandes banques privées et Landesbanken :

$20 \%$ des créances bancaires

Tableau 3 : Principaux comptes de bilan des diverses catégories de banques en RFA (en \% du total)

\begin{tabular}{|c|c|c|c|c|c|c|}
\hline \multirow{3}{*}{ Secteur } & \multicolumn{2}{|c|}{ Total du bilan } & \multicolumn{4}{|c|}{ Actifs } \\
\hline & \multirow[b]{2}{*}{2007} & \multirow[b]{2}{*}{2010} & \multicolumn{2}{|c|}{ Crédits interbancaires } & \multicolumn{2}{|c|}{ Crédits à la clientèle } \\
\hline & & & 2007 & 2010 & 2007 & 2010 \\
\hline Grandes banques commerciales & 18,1 & 25,0 & 18,5 & 20,2 & 16,2 & 13,1 \\
\hline Autres banques privées & 10,5 & 11,2 & 10,8 & 12,4 & 10,8 & 13,2 \\
\hline Sparkassen & 13,9 & 13,0 & 7,9 & 9,1 & 18,9 & 19,2 \\
\hline Landesbanken & 20,0 & 17,5 & 26,8 & 20,3 & 15,5 & 16,8 \\
\hline Coopératives de crédit & 8,3 & 8,4 & 5,2 & 5,6 & 10,7 & 11,8 \\
\hline Org. centraux des coopératives de crédit & 3,3 & 3,1 & 5,6 & 6,6 & 1,5 & 1,4 \\
\hline \multirow[t]{4}{*}{ Ets. à attributions particulières } & 25,8 & 21,7 & 25,2 & 25,8 & 26,5 & 24,4 \\
\hline & \multicolumn{2}{|c|}{ Total du bilan } & \multicolumn{4}{|c|}{ Passifs } \\
\hline & & & \multicolumn{2}{|c|}{ Dépôts interbancaires } & \multicolumn{2}{|c|}{ Dépôts de la clientèle } \\
\hline & 2007 & 2010 & 2007 & 2010 & 2007 & 2010 \\
\hline Grandes banques commerciales & 18,1 & 25,0 & 23,3 & 23,1 & 18,1 & 16,6 \\
\hline Autres banques privées & 10,5 & 11,2 & 13,5 & 14,3 & 12,5 & 16,6 \\
\hline Sparkassen & 13,9 & 13,0 & 10,3 & 9,5 & 24,1 & 23,8 \\
\hline Landesbanken & 20,0 & 17,5 & 25,4 & 20,5 & 12,8 & 12,2 \\
\hline Coopératives de crédit & 8,3 & 8,4 & 3,8 & 5,0 & 15,6 & 16,0 \\
\hline Org. centraux des coopératives de crédit & 3,3 & 3,1 & 6,9 & 6,6 & 1,5 & 1,0 \\
\hline Ets. à attributions particulières & 25,8 & 21,7 & 16,7 & 20,9 & 15,5 & 13,8 \\
\hline
\end{tabular}

Source des données : Deutsche Bundesbank, calculs des auteurs.

A la différence des grandes banques commerciales, les Landesbanken, qui sont le second étage (ou échelon) de l'organisation des caisses d'épargne allemandes, visent à accroître leurs parts aussi sur le marché du crédit à la clien-
Sparkassen et coopératives de crédit, qui dominent dans la banque de détail... 
... ont mieux résisté à la crise

Les Landesbanken restent de droit public, mais sont soumises au droit commun en cas d'insolvabilité

2006 : modification du régime des garanties

tèle. Avec 16,8 \% du crédit aux particuliers et/ou aux entreprises, leur part est supérieure dans ce segment à celle de toutes les grandes banques privées réunies, ainsi que de l'ensemble du secteur coopératif. Durant la crise financière, les grandes banques commerciales avaient vu se réduire de trois points de pourcentage leur part de marché dans le crédit à la clientèle. Du fait que les banques de crédit privées et les Landesbanken (mais aussi les organismes centraux des coopératives de crédit et les établissements à attributions particulières) sont presque exclusivement actives sur le marché des opérations interbancaires, les Sparkassen et les coopératives de crédit sont les seules à miser systématiquement sur le métier classique de la banque de détail. Avec environ $19,2 \%$ des créances non bancaires, les Sparkassen sont plus largement présentes sur le segment du retail banking que les coopératives de crédit $(11,8 \%)$; ces deux catégories y ont même légèrement accru leurs parts de marché entre 2007 et 2010.

Du côté du passif, on observe la prédominance du refinancement par les dépôts de la clientèle privée aussi bien chez les coopératives de crédit que chez les caisses d'épargne allemandes. Et avec $24 \%$ du total des dépôts non bancaires, les Sparkassen sont de loin en première position. Sous l'angle du passif comme de l'actif, les opérations interbancaires jouent un rôle secondaire pour ces deux types d'établissements œuvrant au niveau régional et voués au métier classique d'octroi de crédits.

Lorsqu'on cherche à établir une relation entre les différents modèles économiques et le degré de résistance à la crise financière, on est naturellement tenté d'attester un effet stabilisateur à la poursuite d'activités bancaires classiques, puisque tant les Sparkassen que les coopératives de crédit ont résisté visiblement sans dommages à la transmission de la crise de la finance mondiale au système bancaire allemand.

\section{Modification du cadre réglementaire pour les banques de droit public}

Les différences constatées dans les modèles économiques des diverses catégories de banques ont de surcroît été accrues par les modifications intervenues dans le cadre réglementaire s'appliquant aux établissements de droit public, et tout particulièrement au domaine d'activités des Landesbanken. Jusqu'en 2005, la participation au capital d'un établissement de droit public (Anstalt des öffentlichen Rechts) avait pour corollaire la responsabilité pleine et entière du porteur et garant (Träger). Les Landesbanken profitaient jusque là de la servitude (Anstaltslast) et de la garantie de leur porteur de parts (Gewährträgerhaftung : responsabilité du Träger ; voir Schlierbach/Püttner, 2003, pp. 144-148).

Le principe de servitude (Anstaltslast) désigne l'obligation légale faite à la collectivité fondatrice (Errichtungskörperschaft) de la Landesbank (ou de la Sparkasse) de "veiller au bon fonctionnement de l'établissement durant la totalité de son existence et de remédier à d'éventuelles lacunes dans son financement à l'aide d'allocations compensatoires ou par tout autre moyen approprié » (Bankenenquête, 1968, p. 47). Le Land porteur de parts garantit de la sorte en permanence la solvabilité de la banque concernée. Alors que le principe de servitude s'applique aux liens internes entre le(s) porteur(s) et la Landesbank, celui de la responsabilité du porteur (Gewährträgerhaftung) régit la relation externe entre les porteur(s) de l'établissement et ses créanciers. Le Land concerné est donc garant (il se porte de facto caution en cas de défaut) du passif de la Landesbank, et ce de façon illimitée.

Au cours des années 1990 avait émergé un virulent débat sur les effets de ces deux dispositions sur la concurrence. Aux yeux notamment des établissements de droit privé, l'obligation faite aux Länder de se porter garants des établissements publics s'assimilait à une subvention ouvrant accès aux bénéficiaires à des conditions de refinancement privilégiées sur le marché des capitaux. Et en décembre 1999, la Fédération bancaire de l'UE engagea une procédure for- 
melle pour entrave à la concurrence contre ces deux moyens de cautionnement. La Commission européenne suivit largement l'argumentation de la fédération et diagnostiqua elle aussi une subvention publique illicite. Le compromis auquel parvinrent en juillet 2001 la Commission, le gouvernement fédéral et les établissements de droit public mena à l'abrogation de la responsabilité du porteur et à la modification du principe de la servitude du porteur, transposée dans le droit commun des obligations du propriétaire. Depuis, les Landesbanken continuent certes à relever du statut des établissements de droit public (dont les porteurs de parts sont des collectivités publiques), mais le régime antérieur de garanties ne s'applique plus à elles, de sorte que ces banques publiques sont désormais soumises au même droit que les banques privées en cas d'insolvabilité.

Pour la mise en conformité avec la nouvelle réglementation, Landesbanken et Sparkassen se sont vues accorder des dispositions transitoires. Le passif préexistant n'est ainsi pas concerné par la modification. Les engagements financiers conclus entre le 18 juillet 2001 et le 18 juillet 2005 ne sont couverts par la garantie des porteurs de parts que jusqu'au 31 décembre 2015 (voir Witt, 2006, pp. 18-23). Afin de réduire les retombées négatives liées à l'abrogation de la garantie des porteurs de parts, les banques centrales des Länder ont usé de cette possibilité pour emprunter massivement des capitaux extérieurs. Or le volume de ces capitaux dépassait de loin celui qui était requis pour les opérations actives, de sorte que ce surcroît fut investi dans des placements à risque. Cette 'fuite en avant' vers le marché mondial des capitaux s'observe depuis 2003 dans l'importance croissante des opérations en valeurs mobilières (graphique 3).

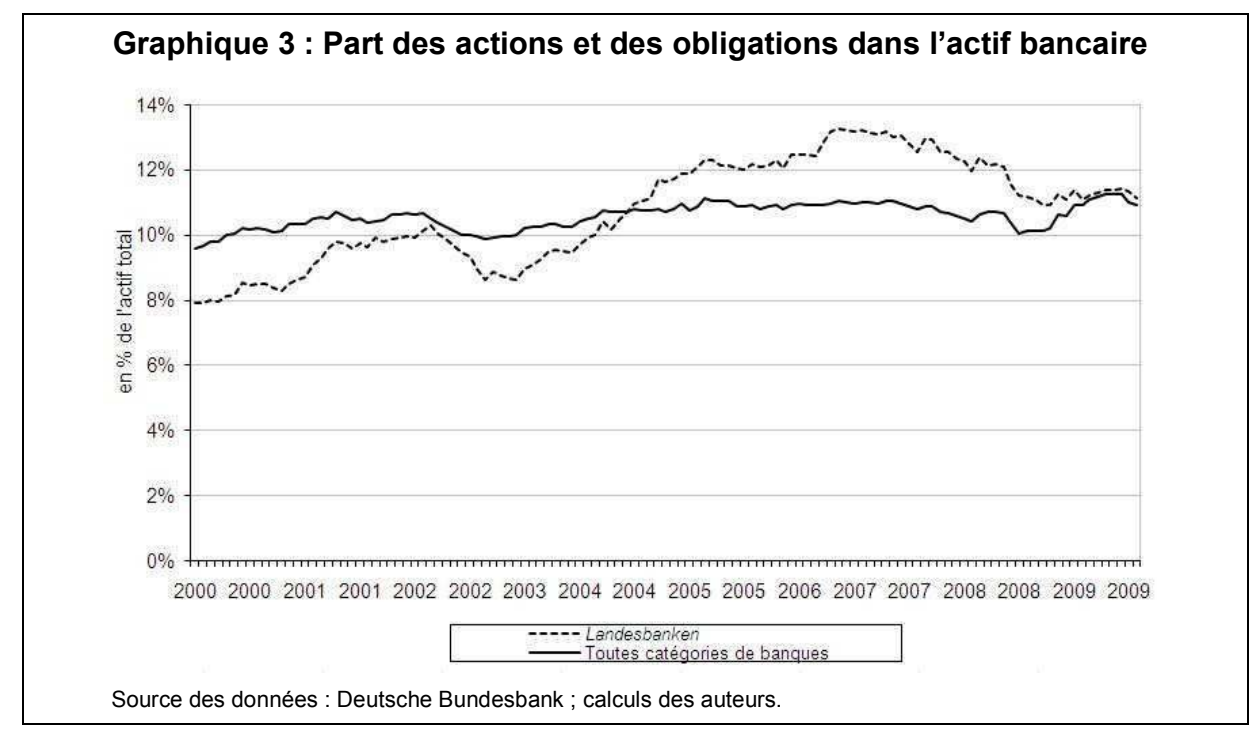

Engagement croissant sur le marché mondial des capitaux

Dans le même temps, les grandes banques commerciales changeaient elles aussi de stratégie à la fin des années 1990. L'examen critique des coûts administratifs et des coûts salariaux d'une part, des résultats comparés réalisés dans les différents segments de marché de l'autre, eut pour conséquence que nombre d'établissements qui œuvraient principalement dans le segment de la banque de crédit abandonnèrent le métier de banque universelle et se retirèrent donc ainsi du segment de la banque de détail, considéré comme trop 'dispersé'. Même l'octroi de crédits aux petites et moyennes entreprises fut considéré de plus en plus sous l'angle de la rentabilité et de moins en moins sous celui de la fidélisation de la clientèle, ce qui eut pour effet de réduire considérablement au fil des années la part des crédits à la clientèle dans l'actif total des banques concernées. Le graphique 4 ci-dessous montre cette évolution depuis 1995. 
Grandes banques privées : depuis dix ans, réorientation sur la banque d'investissement

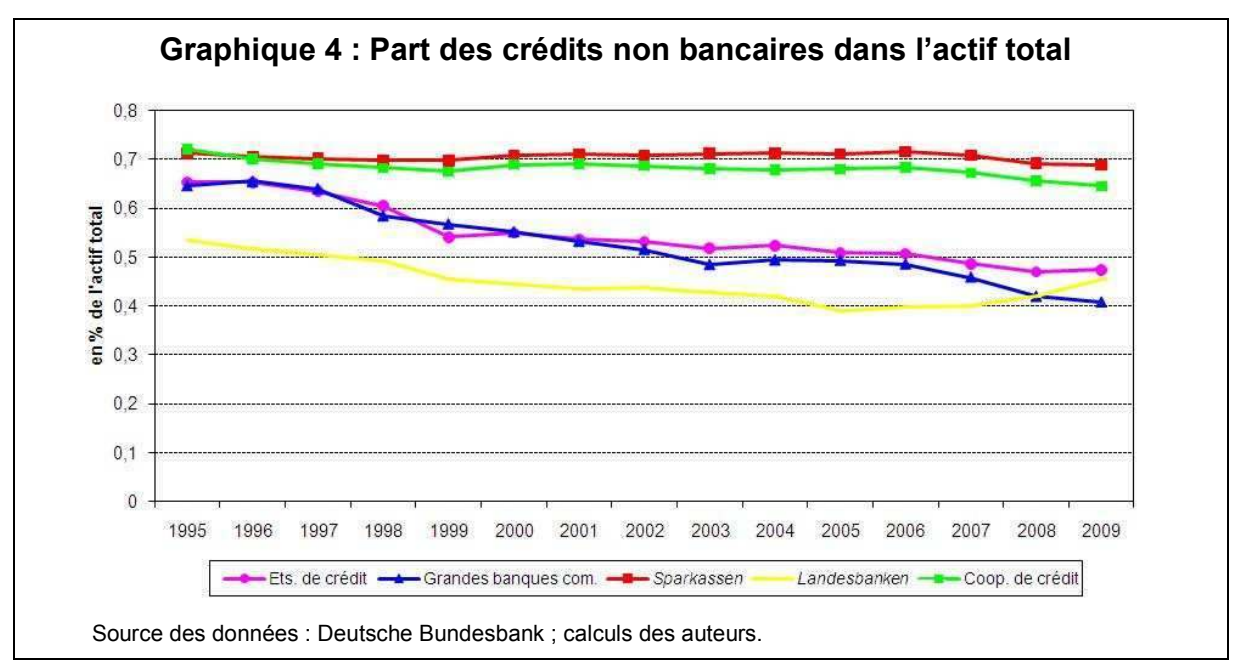

Les grandes banques commerciales surtout se sont repositionnées sur le métier de la banque d'investissement. Elles n'ont donc plus octroyé les crédits directement, mais par le biais d'intermédiaires financiers privés ou institutionnels, par exemple en organisant et gérant les émissions d'actions nouvelles ou les souscriptions d'emprunts, de même qu'en développant systématiquement le segment des commissions sur les transactions mobilières et les opérations de contrepartie. Les grandes banques commerciales, mais aussi certaines Landesbanken, en se transformant ainsi exclusivement en prestataires, ont donc besoin de moins de capitaux propres pour couvrir les risques, dès lors qu'elles ne conservent dans leur portefeuille aucune part des émissions (lancées via leurs intermédiaires). Par ailleurs, le métier de banque d'affaire permet d'éviter les risques liés aux variations des taux d'intérêts et des prix qui sont inhérents aux opérations classiques de crédit, et de liquider à l'inverse les commissions négociées pour les prestations rendues. De ce fait, les excédents du courtage ont (du moins jusqu'à ce qu'éclate la crise de la finance mondiale) une importance nettement supérieure en tant que source de revenus nets pour les banques de crédit et les grandes banques commerciales que pour les Sparkassen ou les coopératives de crédit (voir graphique 5).

\section{Graphique 5 : Part de l'excédent issu du courtage dans l'excédent total}

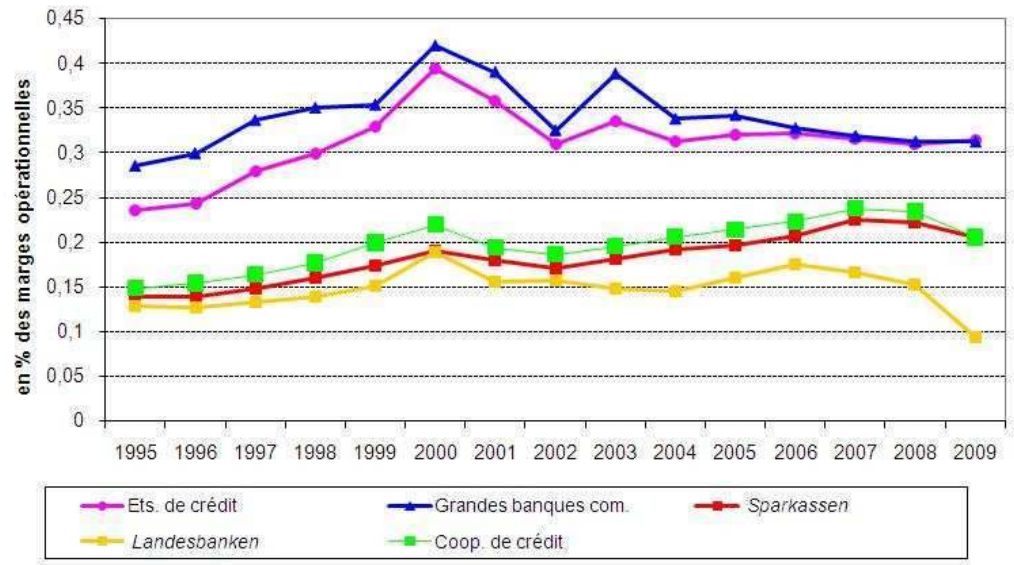

Source des données : Deutsche Bundesbank; calculs des auteurs.

Le secteur public des Sparkassen et des banques coopératives a certes bénéficié des profonds changements observés dans les pratiques de placements de sa clientèle et vu s'accroître considérablement ses activités de courtage, il n'en reste pas moins qu'il réalise toujours quelque $80 \%$ de son excédent grâce aux 
frais financiers des transactions. Chez les grandes banques commerciales par contre, cette part était tombée entre-temps à moins de $60 \%$.

La différenciation des modèles économiques entre d'une part le segment des banques de crédit et les Landesbanken, d'autre part entre les Sparkassen fortement orientées sur le Mittelstand et la clientèle des particuliers, ainsi que les coopératives de crédit, est nettement plus prononcée depuis ces dernières années, ce qui contribue à expliquer les différences constatées dans la résistance des banques allemandes durant la crise de la finance mondiale. Retenons ici en guise de première conclusion que la structure spécifique du système bancaire allemand, souvent taxée d'inefficiente par les observateurs étrangers, présente, principalement du fait de la différenciation de l'offre, une remarquable capacité à absorber les chocs exogènes (voir Gischer, 2010).

\section{Les conséquences de la crise de la finance mondiale pour le secteur bancaire allemand}

Au vu de cette analyse, force est de constater que les effets qu'a eus la crise de la finance mondiale sur le système bancaire allemand sont intimement liés aux modèles économiques différenciés des diverses catégories de banques. Alors que les Sparkassen et les coopératives de crédit n'ont été qu'indirectement affectées par les turbulences sur les marchés mondiaux, les grandes banques commerciales et les Landesbanken ont connu d'importantes mutations structurelles.

Dès 2007, la SachsenLB, la Landesbank de la Saxe, qui était impliquée dans le marché hypothécaire américain par l'intermédiaire de sa filiale ad hoc en Irlande (Ormond Quay), faisait face à d'importantes difficultés de trésorerie et dut être reprise la même année par la LBBW, la Landesbank du Bade-Wurtemberg. De même, après avoir connu une dépréciation abyssale de son portefeuille d'asset backed securities, la Landesbank bavaroise BayernLB, de même que la WestLB (Land de Rhénanie du Nord-Westphalie) et la HSH Nordbank (Länder de Hambourg et de Schleswig-Holstein) ont dû recourir à une hausse de leurs capitaux propres et à la caution du gouvernement fédéral et du gouvernement de leur Land. Par ailleurs, la BayernLB, jusque là actionnaire majoritaire de la SaarLB (Land de Sarre), banque franco-allemande dédiée aux PME, réduisit sa part à son tour de table, de sorte que depuis juin 2010, la SaarLB a retrouvé son autonomie.

Parmi les grandes banques commerciales, la première à tirer profit de la crise fut la Commerzbank AG qui put ainsi absorber à bon compte sa concurrente Dresdner Bank AG dont la capitalisation avait fondu. Mais pour se mettre en difficulté peu après, du fait de la hausse des risques crédit de la Dresdner Bank $A G$, ce qui mena à l'étatisation partielle de la Commerzbank AG. Par ailleurs, au fil de la crise de la finance mondiale, la Deutsche Bank AG absorba sa concurrente Postbank $A G$, ce qui fait qu'aujourd'hui, après la crise, sur les cinq grandes banques commerciales que comptait à l'origine l'Allemagne, il ne reste plus sur le marché que deux établissements privés autonomes (Deutsche Bank AG, UniCreditbank AG) et un sous contrôle de l'Etat (Commerzbank AG).

Ce bref APERÇU du SyStème À trois PILIERS AVANT, PENDANT et APRÈs la crise de la finance mondiale montre qu'à l'évidence, le modèle économique des 'petites' entités que sont les Sparkassen et les coopératives de crédit a déployé des effets stabilisateurs sur la performance et le bon fonctionnement du système bancaire allemand. Et face à ce constat, on ne peut que s'étonner de voir que le monde économique et politique ne cesse de réclamer la consolidation massive de ce groupe d'établissements qui s'est pourtant révélé le plus résistant à la crise de la finance mondiale et qui, parce qu'il est profondément ancré dans l'économie réelle, a contribué à stabiliser le système bancaire allemand (voir Schildbach, 2008).
Différenciation de l'offre : une des raisons de la résistance du système bancaire allemand

Landesbanken : mutations structurelles

Grandes banques privées : deux seulement restent autonomes 
Plus nuancé se révèle le bilan en ce qui concerne les grandes banques commerciales et une partie du secteur des Landesbanken. Ainsi, la crise de la finance mondiale a douloureusement fait apparaître que les stratégies menées pour diverses raisons par ces deux catégories, à savoir le positionnement prioritaire sur un métier de banque d'investissement très générateur de marges de courtage, sont largement plus volatiles et donc nettement moins durables qu'on ne le pensait jusqu'ici. Quant aux Landesbanken, en comparaison de leurs concurrentes de droit privé, elles souffrent d'un profil trop peu marqué dû à leur manque d'indépendance. Dès lors, la question qui se pose est moins celle de savoir s'il est nécessaire de réorganiser ce secteur dans son ensemble tout en révisant son modèle économique (parfois d'une grande complexité), mais plutôt celle de savoir comment procéder à cette réorganisation et quelle nouvelle configuration lui donner.

\section{Encadré 2 : L'avenir de la WestLB}

Le 30 avril 2008, la Commission européenne a autorisé la mise en œuvre des mesures allemandes de stabilisation des marchés financiers pour venir en aide à la WestLB, l'ancien vaisseau amiral du secteur des Landesbanken, qui avait dangereusement pris de la gîte durant la crise de la finance mondiale. Conformément aux dispositions du Traité de l'UE sur les aides publiques, le soutien de l'Etat s'élevait, mesures d'accompagnement comprises, à environ 5 milliards $€$. L'autorisation de ces aides pour le sauvetage de la WestLB, limitées dans le temps, avait pour condition expresse l'obligation de procéder à une vaste restructuration de cette banque de la Rhénanie du Nord-Westphalie pour l'établir sur des bases plus stables. En cas de non-respect de cette disposition ou en l'absence de présentation d'un projet définitif à la mi-avril 2011 au plus tard, les gardiens de la concurrence à Bruxelles peuvent exiger le remboursement de ces aides, ce qui signifierait l'arrêt de mort de la WestLB.

\section{Les projets de restructuration}

Les propriétaires de la WestLB (Bund, Land de Rhénanie du Nord-Westphalie, Sparkassen) ont récemment transmis à la Commission européenne un projet de restructuration avec trois options. La première prévoit la cession de l'établissement dans son entier, la seconde la poursuite de la baisse du volume d'activités, et la troisième propose un modèle de banque en réseau qui équivaudrait à la création d'une banque centrale pour les Sparkassen (voir Drost et al., 2011, p. 34). Aucune des trois options ne semble remporter actuellement la préférence.

L'idée de fusionner la WestLB avec une autre banque, si possible une autre Landesbank, paraît irréalisable de facto. Car en novembre dernier seulement, la tentative de fusionner la WestLB et la BayernLB avait échoué d'une part du fait de la mauvaise santé de la WestLB et des risques résiduels qu'elle présentait, d'autre part du fait de la résistance du monde politique, principalement celle du gouvernement bavarois.

L'option d'une réduction de sa voilure prévoit de diminuer encore d'environ un tiers son total de bilan qui ne s'établit plus actuellement qu'à quelque 220 milliards $€$. Les charges liées à cette option de restructuration qui serait achevée en 2015 devront partiellement être supportées par les propriétaires de la WestLB. Cette option prévoit en outre la séparation de ses activités commerciales en quatre divisions distinctes, réunies sous le toit d'une banque au périmètre réduit, ce qui faciliterait la cession ultérieure de certaines branches d'activité.

Le modèle de banque en réseau préconisé par les Sparkassen vise l'éclatement de la WestLB. Une partie de cette Landesbank pourrait ainsi assurer la fonction de banque centrale pour la centaine de Sparkassen que compte le Land de Rhénanie du Nord-Westphalie, celles-ci apportant les capitaux nécessaires pour en assurer les fonds propres. Toutes les autres activités seraient cédées ou rattachées à la bad bank qui a vu le jour récemment.

Il est impossible cependant de procéder à une révision du modèle économique des Landesbanken qui soit couronnée de succès sur le long terme sans prendre en considération les interdépendances internes au réseau des établissements de droit public. Car ces banques régionales ne sont pas seulement membres à part entière de la 'grande famille' qu'est le secteur des Sparkassen, mais elles sont également (d'anciens) objets de prestige pour leurs co-actionnaires, les Länder. Dès lors, la voie d'une « reconfiguration », déjà semée d'embuches économiques, s'encombre aussi d'obstacles politiques (voir encadré 2). Pour que celle-ci soit réalisable, il convient donc d'envisager des solutions reposant essentiellement sur l'identification de la demande ou des besoins dont la couverture est revendiquée par les Sparkassen et autres établissements rassemblés au sein de l'Association allemande des caisses d'épargne et des Lan- 
desbanken (Deutscher Sparkassen- und Giroverband, DSGV), de même que les clients particuliers, professionnels ou institutionnels, mais qui n'est pas assurée par les autres établissements financiers. En revanche, toutes les tentatives visant à asseoir durablement les Landesbanken au sein de la 'famille' des établissements de droit public dans le rôle d'entités comparables à de grandes banques commerciales peuvent être considérées comme vouées à l'échec. Voilà pourquoi il ne reste à celles qui ont une chance de survie dans le réseau que la solution de renouer avec leurs racines, c'est-à-dire de se recentrer sur leur cœur de métier originel.

Mais même un tel revirement "back to the roots " ne pourra pas, compte tenu des fonctions à redistribuer au sein du groupe Sparkassen-Finanzgruppe, s'effectuer d'un côté sans fusions ni de l'autre sans une externalisation conséquente (c'est-à-dire la cession) de secteurs entiers d'activité. II n'est guère imaginable qu'à l'avenir, une Landesbank de droit public continue à mener sous son toit des activités aussi porteuses de risques que les opérations de crédit de substitution. II est prévisible au contraire que les propriétaires des Landesbanken, rudement mis à mal durant la crise de la finance mondiale, à savoir les fédérations régionales des Sparkassen et surtout leurs membres, veilleront d'euxmêmes à ne pas être entraînés dans une situation engageant leur responsabilité mais dont la maîtrise leur échappe et qu'ils ne peuvent encore moins approuver en termes de stratégie commerciale.

On peut observer d'ores et déjà chez les Landesbanken une tendance à développer des activités d'une plus grande stabilité : ainsi, par exemple, la BayernLB et la LBBW ont entrepris (certes sous la contrainte de la Commission européenne) de développer considérablement leur engagement vis-à-vis du Mittelstand et de réduire leurs activités dans la banque d'investissement. Mais il ne peut s'agir là toutefois que d'un premier pas. Car il est indispensable de créer une instance, composée d'un ou de plusieurs établissements, qui mette à la disposition de chacune des Sparkassen toutes les prestations que celle-ci - du fait de sa petite taille - ne peut offrir elle-même à son seul niveau. II s'agit par exemple de conclure des affaires à l'étranger pour le compte de la clientèle des entreprises ou de mener des opérations sur les marchés des capitaux pour celui des collectivités locales. En complément cette ou ces Landesbank(en) pourrai(en)t également couvrir les champs : grands risques ou financement immobilier commercial ; mais en veillant à y associer en cas de besoin la Sparkasse locale concernée. En aucun cas il ne peut être dans l'intérêt du groupe Sparkassen-Finanzgruppe de réduire sa gamme de prestations et d'abandonner ainsi une partie de ses activités à la concurrence. II n'en reste pas moins qu'il convient de tirer les enseignements de l'expérience désastreuse de la période récente et donc de passer toutes les activités de chacune des Landesbanken au crible d'une analyse critique coûts/avantages en termes de rentabilité.

(Traduction : I. Bourgeois)

\section{Indications bibliographiques}

- Bankenenquete 1968: Bericht der Bundesregierung über die Untersuchung der Wettbewerbsverschiebungen im Kreditgewerbe und über eine Einlagensicherung, in Deutscher Bundestag, 5. Wahlperiode, Drucksache V/3500, Bonn

- Brämer P., Gischer H., Pfingsten A., Richter T. (2010a), « Der öffentliche Auftrag der deutschen Sparkassen aus der Perspektive des Stakeholder-Managements », Zeitschrift für öffentliche und gemeinwirtschaftliche Unternehmen, Vol. 33(4), 2010

- Brämer P., Gischer H., RichteR T. (2010b), «Quo vadis Landesbanken? », in EUROPÄISCHES ZeNTRUM Für FödeRALISMUSFORSCHUNG (ed), Jahrbuch des Föderalismus 2010, Baden-Baden, 2010

- BrÄmer P., Gischer H., Richter T., « Bankensystem und Regulierung - Lehren aus der Finanzkrise», in OBERENDER, P. (ed), Reform der Regulierung der Finanzmärkte - unter wettbewerblichen Aspekten, Schriftenreihe des Vereins für Socialpolitik, Berlin, 2011 
-Brunner, A., Decressin J., HaRdy D., Kudela B., « Germany's Three-Pillar Banking System Cross Country Perspectives in Europe », Occasional Paper No. 233, International Monetary Fund, Washington DC, 2004

-Drost F. M., KöHLeR P., LudWig T., « Brüssel verliert die Geduld » , Handelsblatt, 29-03-2011

- FRANKenBerger W., « Das Regionalprinzip - sinnvolles Ordnungsprinzip oder Teufelszeug ? », in Zeitschrift für das gesamte Kreditwesen, 57. Jg., cahier 19, 2004

- GISCHER H., «Wettbewerb und Effizienz in Bankenmärkten », in WISSENSCHAFTSFÖRDERUNG DER SPARKASSEN-FINANZGRUPPE E.V. (ed), Geschäftspolitische Steuerung - Die Sparkassen zwischen Renditeorientierung und Gemeinwohl, Stuttgart, 2010

- Gischer H., Herz B., Menkhoff L., Geld, Kredit und Banken, Berlin, 2005 (2 ${ }^{\text {ème édition) }}$

- Hackethal A., Schmidt R. H., "Structural Change in the German Banking System ? », Revue d'Economie Financière, Vol. 78, 2005

- Hartmann-Wendels T., Pfingsten A., Weber M., Bankbetriebslehre, Heidelberg, 2010 (5 édition)

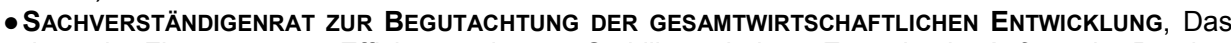
deutsche Finanzsystem : Effizienz steigern - Stabilität erhöhen, Expertise im Auftrag der Bundesregierung, 2008

- SChackmanN-Fallis K.-P., Weiß M., « Landesbanken und Sparkassen - Die Zukunft des öffentlich-rechtlichen Bankensektors », in STIFTUNG GESELLSCHAFT FÜR RECHTSPOLITIK, TRIER, et INSTITUT FÜR RECHTSPOLITIK AN DER UNIVERSITÄT TRIER (eds), Bitburger Gespräche : Jahrbuch 2009/I. Neuordnung der Finanzmärkte : Von der Krise zur Reform, Munich, 2010

- Schildbach J., «European banks: The silent (r)evolution », in Deutsche Bank Research, EUMonitor, Finanzmarkt Spezial, $n^{\circ} 54$, Francfort/Main

- Schlierbach H., PüttNer G., Das Sparkassenrecht in der Bundesrepublik Deutschland, Stuttgart: Deutscher Sparkassenverlag, 2003 ( $5^{\text {ème }}$ édition)

- Stiele M., Wettbewerb im Bankensektor, Wiesbaden, 2008

- WitT C., Bewertung von öffentlich-rechtlichen Sparkassen im Rahmen einer Privatisierungsentscheidung, Wiesbaden, 2006. 